\section{$(3)$ \\ HORIZONTES}

www.revistahorizontes.org

\title{
Modelo de supervisión educativa para dinamizar instituciones educativas hacia una educación de calidad
}

\author{
Educational supervision model to dynamize educational institutions towards quality \\ education
}

Modelo de supervisão educacional para dinamizar instituições educacionais em direção a uma educação de qualidade

\author{
José Luis Olivo-Franco \\ joseolivofranci@hotmail.com \\ Universidad Pedagógica Experimental el Libertador (UPEL), Venezuela, \\ Docente en Institución Educativa Técnica Agrícola Juan Domínguez Romero. Colombia
}

Recibido abril 2020 | Revisado mayo 2020 | Publicado en julio 2020

\section{RESUMEN}

El presente artículo desarrolló un análisis sobre la representación y formas de concebir el rol de los supervisores educativos por parte de los docentes del municipio de Malambo (Colombia), así como una consideración teórica sobre los replanteamientos que demanda la labor del supervisor educativo, a fin de que sea posible responder a los desafíos inherentes a las metas de una educación de calidad planteadas por la UNESCO, la OEI (Organización De Los Estados Iberoamericanos), y el gobierno Colombiano. Para ello, en primer lugar se describen las impresiones de un grupo de docentes de este municipio con respecto al rol que desempeñan sus respectivos supervisores educativos en el proceso de mejora educativa institucional, para finalmente promover una resignificación, como lo hacen otros autores del modelo de supervisión tradicional hacia uno que sea coherente con principios como la integralidad, que intente responder a los retos actuales en el campo educativo.

Palabras clave: Supervisión educativa, gestión educativa, calidad educativa,

\section{ABSTRACT}

This article developed an analysis on the representation and ways of conceiving the role of educational supervisors by teachers in the municipality of Malambo (Colombia), as well as a theoretical consideration on the rethinking that the work of the educational supervisor demands, in order that it is possible to respond to the challenges inherent in the goals of quality education set by UNESCO, the OEI (Organization of IberoAmerican States), and the Colombian government. For this, firstly, the impressions of a group of teachers from this municipality regarding the role played by their respective educational supervisors in the process of institutional educational improvement are described, to finally promote a resignification, as other authors of the Traditional supervision towards one that is consistent with principles such as comprehensiveness, that tries to respond to current challenges in the educational field.

Key words: Educational supervision, educational management, educational quality, qualitative research 


\section{RESUMO}

Este artigo desenvolveu uma análise sobre a representação e formas de conceber o papel dos supervisores educacionais por professores do município de Malambo (Colômbia), bem como uma consideração teórica sobre o repensar que o trabalho do supervisor educacional exige, a fim de que é possível responder aos desafios inerentes às metas de educação de qualidade estabelecidas pela UNESCO, pela OEI (Organização dos Estados Ibero-Americanos) e pelo governo colombiano. Para isso, primeiramente, são descritas as impressões de um grupo de professores desse município sobre o papel desempenhado por seus respectivos supervisores educacionais no processo de aprimoramento educacional institucional, para finalmente promover uma ressignificação, como outros autores da Supervisão tradicional em direção a uma que seja consistente com princípios como a abrangência, que tente responder aos desafios atuais no campo educacional.

Palavras-chave: Supervisão educacional, gestão educacional, qualidade educacional, pesquisa qualitativa

\section{INTRODUCCIÓN}

Desde hace tiempo es común toparse en el campo de la educación con un tema pendiente $\mathrm{y}$ trascendental en todas las agendas de los gobiernos latinoamericanos y del mundo a saber, el asunto de la calidad (Organisation for Economic Co-operation and Development [OECD] 2016; UNESCO, 2014). Al respecto, Metas educativas 2021 suscita a una profunda resignificación del rol de los supervisores en ese cometido (Organización de los Estados Iberoamericanos, 2010). Y es inevitable si se habla de supervisión educativa plantearse desde el principio serios interrogantes cuyas respuestas tengan en miras contribuir a tan anhelado propósito. Cuestiones tales como por ejemplo: ¿Cómo puede la supervisión educativa y los supervisores educativos convertirse en agentes de cambio educativo hacia el alcance de la calidad?, ¿Qué replanteamientos es necesario efectuar desde el o los modelos de supervisión educativa a fin de lograr tales metas? La reflexión sobre estos interrogantes es el propósito central del presente artículo.

\section{TEORIAS REFERENCIALES}

Desde un marco epistemológico es ineludible señalar a los supuestos y principios teórico-conceptuales de la supervisión desde una concepción de ciencia abarcadora y no desde la concepción positivista (García, 2002; Martínez, 2012). Ello permite hablar de principios y de una teoría unificadora de la supervisión desde la ciencia de la administración y la dirección (Mogollón, 2004; Tamayo y Valiente, 2019).De hecho, García (2002), considera la administración como una ciencia cuyo fin es el estudio de las leyes y principios regentes del proceso consciente $y$ continuo de cualificación sistémica de una organización.

En este mismo hilo argumental la dirección como ciencia atribuye a la supervisión el significado de un fenómeno complejo. Sin embargo y pese a la poca literatura existente en calidad de generalizaciones a manera de principios y teorías relacionadas con la supervisión García (2002), enuncia un sistema de principios conceptuales de la administración que en cualquier caso pueden guiar el comportamiento de las organizaciones, incluyendo por supuesto las educativas. Por otro lado, Valle et al. (2006), proponen dentro del contexto escolar: a) el principio de la unidad de la centralización y descentralización, b) el principio del aumento de participación activa $y$ democrática, c) el principio del eslabón fundamental, y d) el principio de la consideración de la unidad en la diversidad y viceversa. En la matriz 1, se ha sintetizado algunos autores y sus respectivos aportes relacionados con la gestión y la supervisión educativa y que se han constituido las bases teórico-conceptuales de los modelos de gestión y supervisión en el ámbito educativo. 
Matriz 1. Algunos aportes de autores sobre gestión y supervisión educativa.

\begin{tabular}{llr}
\hline Autor/es & \multicolumn{2}{c}{ Aportes } \\
Rodríguez (1948) & Reconoce que los \\
& autores se esfuerzan \\
& por validar los \\
& fundamentos & \\
& científicos de la \\
inspección escolar & \\
Kaufman (1974) & Teoría de los enfoques
\end{tabular}

Chadwick (1992);

García (1963), Franseth, Abordan la supervisión (1967); Nérici, (1975); educativa y permiten Fermín, (1980); Alvarado, establecer normas de (1990); Guarderas, (1993); gestión, teóricas y Soler, (2015); Barrasco y metodológicas de Suárez, (2007); Secadura, ayuda práctica. (2008); De Sousa, (2005); Fayol, (1977); entre otros. Stufflebeam (1996).

García, Valle, y Ferrer (1996)

Casassus (2000)

Bertanlaffy (2000) Principios del cambio educativo

Sustentan el modelo de supervisión educativa

Sustenta el modelo de supervisión educativa

Propuesta evolutiva e histórica de los modelos de gestión

Teoría general de los sistemas de Bertanlaffy

\section{Descripción}

Validación de autores de fundamentos

científicos de la inspección educativa

Presenta varias etapas para identificar problemas basados en las necesidades, las cuales favorecen el éxito en los procesos administrativos

Con su enfoque contribuye las herramientas y conceptos a que desarrollen en la organización escolar la totalidad de un sistema, permitiendo su funcionamiento como institución.

Señalan en conjunto normas teóricometodológicas que sirven de guía práctica.

Proponen un modelo de supervisó educativa que integra cuatro fases: a) contexto $y$ necesidades, b) Entrada (Input), que comprende la categorización de las funciones del supervisor en el ámbito escolar; c) Acción supervisora: la cual está integrada por: planificar, organizar, orientar, dirigir, ejecutar, coordinar, supervisar y evaluar; y d) evaluación del proceso de supervisión.

-Proceso del incremento de la participación activa y democrática

-Proceso del cambio de significado de la actividad.

-Proceso del desarrollo y estimulación de la creatividad.

-Proceso de la motivación.

Propone modelos normativo, prospectivo, estratégico, estratégico-situacional, de calidad total, de reingeniería y comunicacional.

Aporta definiciones exactas de conceptos al mismo tiempo las somete a análisis cuantitativo evitando malos entendidos donde se expliquen los fenómenos observables insertados en el sistema de supervisión escolar. 


\begin{tabular}{|c|c|c|}
\hline Autor/es & Aportes & Descripción \\
\hline Pozner (2000) & $\begin{array}{l}\text { Desde la teoría } \\
\text { organizacional estudia } \\
\text { la gestión educativa } \\
\text { desde la complejidad } \\
\text { del contexto escolar }\end{array}$ & $\begin{array}{l}\text { Propone tres niveles de gestión en } \\
\text { educación: la gestión institucional educativa } \\
\text { (macro), la gestión escolar (meso), y la } \\
\text { gestión pedagógica (micro). }\end{array}$ \\
\hline Chiavenato (2000) & $\begin{array}{l}\text { Propone las Funciones } \\
\text { Universales de la } \\
\text { Administración }\end{array}$ & $\begin{array}{l}\text { Las funciones universales de la } \\
\text { administración, están relacionadas con las } \\
\text { actividades que ejecutan los supervisores. }\end{array}$ \\
\hline García (2002) & $\begin{array}{l}\text { Principios teórico- } \\
\text { conceptuales que rigen } \\
\text { la administración de las } \\
\text { organizaciones }\end{array}$ & $\begin{array}{l}\text {-Principio de la objetividad } \\
\text {-Principio de la autonomía } \\
\text {-Principio de la interacción humana } \\
\text {-Principio de la tendencia a la satisfacción de } \\
\text { las necesidades } \\
\text {-Principio de orientación a la cualidad } \\
\text { sistémica } \\
\text {-Principio de la mejora } \\
\text {-Principio de la situación limite } \\
\text {-Principio de la restricción } \\
\text {-Principio de la conectividad } \\
\text {-Principio de la unidad de administración y } \\
\text { la información } \\
\text {-Principio de la medición. }\end{array}$ \\
\hline Bush (2006) & $\begin{array}{l}\text { Teoría de Educational } \\
\text { Management }\end{array}$ & $\begin{array}{l}\text { Su teoría organizacional de la educación } \\
\text { enfatizan en las funciones comunicacionales, } \\
\text { de la ambigüedad, colegiadas, políticas, } \\
\text { subjetivas y de la cultura organizacional. }\end{array}$ \\
\hline Valle et al (2006) & $\begin{array}{l}\text { Principios en el ámbito } \\
\text { de gestión escolar }\end{array}$ & $\begin{array}{l}\text {-Principio de la unidad de centralización y } \\
\text { descentralización. } \\
\text {-Principio del aumento de la participación } \\
\text { activa y democrática } \\
\text { Principio del eslabón fundamental } \\
\text {-Principio de la consideración de la unidad } \\
\text { en la diversidad y viceversa. }\end{array}$ \\
\hline
\end{tabular}

De otro lado es conveniente explicitar que se entiende por supervisión educativa desde las perspectivas de este estudio. Al respecto, hay que reconocer, tal como lo hace Moyano, (2011), que la supervisión "es un concepto complejo" (p.1). Si bien, un primer acercamiento a su significado lleva a relacionar la supervisión con sinónimos como "controlar, inspeccionar, comprobar y revisar" (p.1). Es urgente revisar las concepciones que limitan la supervisión desde un paradigma que solo enfoca aspectos administrativos (Tamayo y Valiente, 2019).

De esta manera encontramos definiciones como la aportada por García (2015), "la supervisión educativa tiene una gran relevancia dentro del sistema ya que sirve de agente que impulsa el dinamismo, la interactividad y retroalimentación constante dentro de la educación"(p. 132).

Ahora bien, aunque no se hará un recorrido detallado por todas las significaciones halladas en la bibliografía, si se precisa hacer mención de que aunque supervisión e inspección son conceptos relacionados, subyace una marcada diferencia entre uno y otro. En el caso de la inspección, como práctica es inicialmente concebida como una forma fiscalizadora del trabajo de los maestros, una institución de control y vigilancia administrativa, fundamentada en el poder del estado. (Moyano, 2011, p. 2). 
Por otro lado, la supervisión desde una concepción académica es la función escolar que propende a la mejora de la educación mediante la asistencia directa a los docentes, al desarrollo del currículo, al desarrollo del personal y al desarrollo de los grupos. (Glickman, 1990; Topete, Chávez, y García; s.f). Por consiguiente, la supervisión, trasciende o debería trascender la acción fiscalizadora de la inspección, inclinándose hacia el asesoramiento, la guía y la orientación, lo que le confiere un matiz dinámico eminentemente de una práctica social como la educación (Moyano, 2011; Soler, 2001).

No obstante, la práctica de la supervisión educativa sigue estando alejada de interacciones pedagógicas significativas entre los supervisores y rectores, y docentes en parte, como lo reconocen Díaz, García y Legañoa (2018), y Tamayo y Valiente (2019), debido a la falta de proyección consensuada y contextualizada.

Es oportuno reconocer un hecho importante con respecto a la calidad educativa, recordado por Martínez y Hernández (2014), y es que para ir en pos de esta "la supervisión educativa debe hacer énfasis en un aspecto fundamental: su esencia pedagógica; al armonizar las tendencias, unificar criterios sin unificar la actuación y establecer una convergencia de esfuerzos de todos los actores educativos" (p. 86). Es decir, no se debe perder de vista las múltiples implicaciones en una educación de calidad y a no desconocer la atención integral y armónica de las condiciones que ella demanda.

No obstante, dar respuesta a la cuestión anteriormente planteada es válido porque tal como lo reconocen varios autores la supervisión es uno de esos múltiples factores implicados en lograr una mejora educativa (Guerra, 2011; Martínez y Hernández, 2014). Sin embargo, para que ello sea cierto, la realidad es que se debe replantear la mirada que se hace de la supervisión que se organiza entorno del campo educativo.

\section{METODOLOGÍA}

Se desarrolló una investigación desde un paradigma interpretativo/cualitativo basándose en la premisa de que el enfoque cualitativo posibilita tal como lo señala Sánchez, (2013, p.95) "el acceso al simbolismo subjetivo de las acciones (en este caso de los supervisores) tiene para los protagonistas" (los docentes). Por consiguiente, en esta investigación se enfocan la atención en el fenómeno particular de cómo los supervisores educativos del municipio de Malambo, son concebidos desde su rol por los docentes y directivos de las instituciones educativas. Así pues este develar y búsqueda de comprensión de las concepciones idiosincráticas de los docentes define el estudio como una investigación de carácter fenomenológico (Husserl, 1974; Fernández, Hernández y Baptista 2010; Martínez, 2012; Rojas, 2014).

Vale decir que esta realidad particular tal y como la interpretan los sujetos ha sido captada mediante entrevistas, charlas realizadas a un grupo significativo de diez docentes de tres instituciones educativas del municipio de Malambo, incluyendo entres estos, una tutora del programa Todos a Aprender (Ministerio de Educación Nacional 2012), y nueve docentes todos pertenecientes al sector oficial. Este grupo constituye una muestra teórica estructural no estadística suficiente desde los postulados del paradigma post-positivista para lograr la saturación teórica, es decir los patrones recurrentes que posibilitaron la configuración de unidades categoriales que permitieron la aproximación a la comprensión del fenómeno desde los docentes participantes del estudio (Strauss y Corbin, 2002; Teppa, 2012; Vasilachis, 2006). 
Se efectuó un microanálisis línea por línea de los comentarios aportados por los docentes obtenidos a partir de las entrevistas a profundad efectuadas. Se codificaron estos mediantes símbolos alfanuméricos de esta manera por ejemplo, DOC01, en donde los literales indican la palabra docente y los números atendiendo al orden en que se desarrollaron, estos códigos se usan en el presente artículo para referirse a los participantes. Mediante la cromatización esto es con colores diferentes, se identificaron y fragmentaron las ideas de mayor significado y relacionadas con aspectos diferentes (Coffey y Atkison, 2003).

A partir de la información obtenida se logró categorizar las concepciones que tienen los docentes sobre la supervisión educativa que sobre ellos se ejerce y mediante la reflexión desarrollada a partir de distintos autores se han propuesto aspectos en los cuales es necesario replantear no solo el rol del supervisor, sino el modelo de supervisión en el municipio de Malambo.

\section{HALLAZGOS}

Es este aparte, es prudente abordar la exposición de la información recolectada mediantes estrategias y técnicas como charlas $\mathrm{y}$ entrevistas propias de un estudio interpretativo o cualitativo.

El análisis de la información recabada hizo posible una aproximación hacia la representación que tienen estos docentes del Malambo, acerca de la función del supervisor educativo en la actualidad. De tal manera que emergieron a partir de lo aportado por los sujetos participantes dos importantes categorías nominadas basándose en la literatura y constructos teóricos revisados con anterioridad. que al hacer una contrastación no forzada con planteamientos de algunos autores que abordaron aspectos similares en contextos mucho más abarcadores tales como Cortes y Lorente (2013), González, (2007); Miranda, (2002), entre otros, se perciben algunas hallazgos convergentes.

Vale decir que con base a la información recabada la dos categorías constituidas se constituyen a la vez en grandes dificultades o desafíos que han de superarse desde la supervisión educativa para una verdadera participación de los supervisores en la mejora de la calidad educativa, siendo estas:1) Burocratización del trabajo, y 2) Tendencia hacia la instrumentalización. A continuación no solo se describen estos resultados, sino se ofrece una aproximación a su interpretación desde las subjetividades de los docentes, pero también desde un diálogo a la manera de Maturana (2001), con los autores y estudios considerados.

\section{Burocratización del trabajo}

Con relación a la primera categoría emergida, Burocratización del trabajo, se pudo interpretar que en efecto la mayor parte de los docentes expresan que la supervisión en el municipio, se inclina hacia las funciones de control por parte de los supervisores, y cuyas visitas esporádicas son generalmente:

DOC1: "para atender quejas de padres de familia o cuando alguna situación se ha salido de las manos al rector".

DOC2: "solo cuando hay una situación o queja de los padres".

DOC5: "Ahora los padres andan quejándose en secretaria de educación por todo, no saben cuál es el protocolo a seguir, por ejemplo deberían primero abordar si hay un problema con un maestro en particular con ese maestro y eh luego con el rector y así. Pero ahora se van es directamente allá" 
D0C7: "generalmente no vienen mucho aquí, no hacen muchas visitas a la escuela".

En este sentido, un primer obstáculo a superar lo constituye entonces el hecho de la burocratización del trabajo del supervisor, por el predominio de la función de control, asociada a la idea de control del Estado. De tal manera que predomina no solo en Colombia sino en Latinoamérica, tal como lo afirman Cortes, y Lorente, (2013), un modelo de supervisión que privilegia las tareas de control y de índole administrativas sobre el asesoramiento pedagógico, apoyo a los docentes y directivos.

Precisamente son esas mismas funciones tecno-burocráticas las que impiden que los supervisores educativos cumplan con su papel de asesoría y orientación de los centros educativos. Tanto complejo, como preocupante es este hecho que en el estudio realizado por Cortes, y Lorente (2013), reconocen que aspectos fundamentales como la función de ayuda, asesoría e incluso las de control no se pueden ejercer con calidad debido a la saturación en labores como la "entrega de documentos, la solución de quejas de padres, maestros y alumnos, entre otras" (p.2).

En efecto, la situación de la supervisión educativa en Malambo, Colombia, no es muy diferente a la del resto de países iberoamericanos expuesta en el estudio de Cortes, y Lorente, (2013). De hecho, en registros que se hicieron a las pocas visitas que realizaron los supervisores de núcleo, a sus respectivos centros educativos en el municipio de Malambo, para el año 20162017, se evidenció que el motivo de estas era para arreglar situaciones de conflicto, quejas o asuntos de documentación, en ninguno de los casos fue para prestar asesoramiento $\mathrm{u}$ orientación pedagógica que contribuyera en la práctica a la mejora educativa de las instituciones.

Ahora bien, aunque en el plan operativo de apoyo y mejoramiento año 2016-2019, que diseñó la secretaria municipal de Malambo, dentro de las acciones planificadas en este, se proyectó como uno de sus objetivos "Fortalecer internamente la institucionalidad relacionada con el sector educativo, para coordinar, apoyar, y supervisar la prestación del servicio educativo en el territorio municipal" y se menciona un plan de capacitación, asesoría y acompañamiento a los Supervisores de Educación en indicadores de gestión educativa y pedagógica, no se evidenciaron cambios frente a la labor de los supervisores educativos.

\section{Tendencia a la instrumentalización}

Un segundo aspecto, al que dar atención es la instrumentalización de los supervisores educativos por parte del Estado: "El que viene a mirar si todo está en regla" (ED10), señaló jocosamente un docente refiriéndose a que los supervisores visitan en cierto momento del año escolar para requerir la documentación. En la misma línea de opinión otro agrega: "Vienen es a mirar que cosa anda mal, a poner a llenar uno papeles y más papeles" (ED09). Estos papeles se interpretan hacen alusión a documentación requerida por el Ministerio de Educación Nacional [MEN], tales como el Proyecto Educativo Institucional [PEI], Manual de Convivencia, planes de áreas, entre otros, común a este comentario se percibe la opinión de la totalidad de los docentes participantes.

Y es que a través de los supervisores el estado garantiza la vigilancia del cumplimiento de políticas públicas, normativas ministeriales o de los proyectos de reformas educativas emanadas. Aunque esta labor corresponde a una de las funciones del supervisor el hecho de que gran parte de tiempo de trabajo se 
dedique a ser un instrumento de control de los poderes públicos conlleva muchas veces a no operar con mayor flexibilidad en su relación con las instituciones educativas.

\section{REFLEXIONES FINALES}

Luego de esbozar el análisis sobre la caracterización de la función y representación de los supervisores y la supervisión en el municipio de Malambo, visión que al contrastarse sin forzar los datos recolectados, está en consonancia con los estudios realizados por investigadores en el contexto iberoamericano.

Llega el momento para la reflexión impostergable en este trabajo, sobre cómo puede la supervisión educativa y por consiguiente, los supervisores educativos ser agentes dinamizadores hacia la calidad educativa. Para ello, vale reconocer un hecho importante con respecto a la calidad educativa, recordado por Orozco (2009), y es que no se debe perder de vista las múltiples implicaciones en una educación de calidad y a no desconocer la atención integral y armónica de las condiciones que ella demanda.

No obstante, dar respuesta a las cuestión anteriormente planteada es válido porque tal como lo reconocen varios autores la supervisión es uno de esos múltiples factores implicados en lograr una mejora educativa (González, 2007; Miranda, 2002). Sin embargo, para que ello sea cierto, la realidad es que se debe replantear la mirada que se hace de la supervisión que se organiza entorno del campo educativo.

Llegado a este punto ha de plantearse la cuestión de ¿Cómo pueden lo supervisores educativos dinamizar desde su rol a las instituciones educativas, maestros y directivos a su cargo, hacia la calidad educativa en Colombia? Para ello sin duda es esencial en primer lugar que los propios supervisores se apropien y reconozcan que pueden ser agentes de cambio en los diferentes sistemas educativas (Cortes, y Lorente 2013; Fullan 2003; Miranda 2002). Es decir, los supervisores deben replantearse como desde los respectivos contextos escolares socioeducativos pueden mejorar la calidad, en participación y la autonomía de las instituciones educativas.

Este replanteamiento debe hacerse desde la concepción de un modelo de supervisión educativa que optimice las funciones de los supervisores como un proceso pedagógico integral hacia la redefinición de los procesos educativos que finalmente apunten hacia una educación de calidad.

En armonía con ello, se ha considerado los planteamientos de González (2007), en el que articula un modelo de supervisión escolar a la gestión supervisora de forma integrada a los procesos técnicos, administrativos, sociales, de asistencia y de mediación o asesoramiento. En un primer esbozo, vale decir que la categorización de la Gestión Supervisora fue clasificada por Nérici (1975), como funciones: a) técnicas, b) administrativas, y c) sociales; y posteriormente Teixidó (1997), replantea las funciones sociales por de asistencia.

Sin embargo es Vélez (2001), quien aporta al presente estudio un aspecto interesante al agregar a la gestión supervisora la conceptuación de mediación. Es decir, la gestión supervisora desempeña una acción integradora y se categoriza como una acción de Asesoramiento Pedagógico Integral relacionadas tal como las cita textualmente González (2007), con las responsabilidades de: "elegir la solución adecuada, coordinar el éxito docente, resolver los problemas , demostrar eficacia en el ejercicio de las tareas encomendadas, asesorar proyectos, buscar la integralidad comunitaria, además de planificar, orientar, dirigir, ejecutar, 
coordinar, supervisar y evaluar" (p.15). Todas estas funciones van dirigidas finalmente a verificar el cumplimiento de los objetivos y fines de la educación, pero de una forma participativa, integral, cooperativa, competitiva y efectiva.

Un modelo de gestión supervisora Humanista y Social que contemple el Asesoramiento Pedagógico Integral contrasta como se ha de notar, con el modelo de inspección y eficientista, que promulga una gestión supervisora técnica basada solo en criterios de competencia, eficacia, vocación directiva, tecno- administrativa, y que pretende controlar a través del rendimiento de cuentas frecuente (Arriaga, 1996; Topete, Chávez y Márquez; s.f.).

Por consiguiente, la acción supervisora posibilitará optimizar el funcionamiento de las instituciones, no solo los procesos administrativos sino, mejorar la calidad de la educación considerando todos los ámbitos donde se desarrolla la supervisión y en especial el asesoramiento pedagógico.

Igualmente con respecto a las competencias y características del supervisor escolar vale afirmar que este debe contar con un perfil nuevo diferente al expuesto en líneas anteriores. Así pues, una de las principales competencias del supervisor será impulsar, a través de un liderazgo eficaz, el trabajo académico, los proyectos pedagógicos. Haciendo claridad que los procesos administrativos fijos o de rigor no deben afectar el desarrollo de los procesos académicos.

Aquí es conveniente por ultimo dar a conocer que en el estado colombiano apuntando hacia la meta de la calidad educativa lanzó desde hace algunos años el Programa para la Transformación de la Calidad Educativa [PTCE] (MEN, 2012), enmarcado a su vez en las metas del plan de desarrollo, presentado en junio de 2011, plantean como una de las estrategia para la consecución de sus metas el acompañamiento al colectivo de maestros de tutores seleccionados entre los mejores maestros de cada región y que han sido formados para tal acompañamientos.

El tutor del PTCE, se define como un docente que hace el acompañamiento formativo a los docentes de los establecimientos educativos seleccionados para brindarles oportunidades de mejorar sus prácticas de aula, en un ambiente de formación e intercambio de conocimientos, actitudes y buenas prácticas, con la puesta en marcha y mejoramiento de los ambientes de aprendizaje efectivos en contextos especialmente difíciles (MEN, 2012).

A su vez las funciones del tutor se enmarcan en tres grandes categorías: formación, acompañamiento y evaluación. Ahora bien, se destaca al haber desarrolladas estas consideraciones es el hecho de que la función de asesoramiento que corresponde a los supervisores educativos está siendo suplida por estos docentes tutores.

Sería interesante y provechoso adelantar investigaciones que caractericen la representación que tienen los docentes del municipio sobre estos docentes tutores y más importante dar cuenta de la incidencia e impacto que tiene su rol en el proceso de mejoramiento de la calidad educativa en las instituciones del municipio, urgen por tanto, investigaciones desde perspectivas cuantitativas y cualitativas que den a conocer tales aspectos, lo cual constituye continuidad del presente estudio.

\section{REFERENCIAS}

Alvarado, O. (1990). Supervisión Educativa; Teoría y Práctica. Lima, Perú. Educativa: INIDE

Arriaga, R. (1996). Sentido y realidad de la supervisión escolar en educación primaria: El punto de vista del supervisor y del docente. México: tesis de Maestría. UDLA 
Barrasco, L; y Suarez, C. (2007). Supervisión educativa. Actualidad y perspectiva en el mejoramiento de la calidad de los sistemas educativos. Curso 9. Congreso internacional Pedagogía 2007, 1-24. La Habana: Educación cubana. Recuperado de http://scielo.sld.cu/scielo.php?script= sci_nlinks\&pid=S2077-

2955201900010012900005\&lng=en

Bush, T. (2006). Theories of educational management. Recuperado de http://cnx.org/content/m13867/latest/

Casassus, J. (2000). Problemas de la gestión educativa en América Latina. La tensión entre los programas del tipo A y el tipo B. Santiago de Chile, UNESCO. Recuperado de http://www.lie.upn.mx/docs/ Especializa-cion/Gestion/Lec2\%20.pdf

Chadwick, C. (1992). Tecnología educacional para el docente. Madrid, España 3era ed. Editorial Paidós, Ibérica, S.A

Chiavenato, I. (2000). Introducción a la teoría general de la administración. México: 5ta Ed. Editorial Mc Graw-Hill/ Interamericana de México, S.A. de C.V

Coffey, A; y Atkison, P. (2003). Encontrar el sentido a los datos cualitativos. Estrategias complementarias de investigación. Medellín: Editorial Antioquia

Cortes, R. y Lorente, A. (2013). La Supervisión educativa en América Latina ante las metas educativas de 2021, propuestas por la OEI. Revista Iberoamericana de Educación. Recuperado de http://www. rieoei.org/deloslectores/4601Cortes.pdf

De Sousa, M. (2005). Una propuesta de modelo de gestión democrático participativa en el Centro Federal De Educación Tecnológica de Maranhã. Tesis de doctorado inédita. La Habana, Cuba: Instituto Central de Ciencias Pedagógicas. Recuperado de http://www.redem.org/boletin/files/Mad alena_Martins

Díaz, M; García, J; y Legañoa, M. (2018). Modelo de gestión de acompañamiento pedagógico para maestros de primaria. Transformación, 14(1), 44-57. Recuperado de http://scielo.sld.cu/pdf/trf/v14n1/ trf05118.pdf

Fermín, M. (1980). Tecnología de la supervisión docente. Buenos Aires:
Kapalusz

Fayol. H. (1977). Administración industrial y general. México: Herrera Hermanos

Franseth, J. (1967). Supervisión escolar como guía. México D. F.: Trillas

Fullan, M. (2003). Las fuerzas del cambio con creces. Madrid: Akal

García, G. (2002). Contribución a la reorganización del campo científico de la administración: una propuesta epistemológica. Tesis doctoral inédita. La Habana, Cuba: Instituto Superior Politécnico José Antonio Echeverría. Recuperado de https://hera.ugr.es/ tesisugr/26422785.pdf

García, B. (2015). La supervisión educativa ¿víctima o excluida de las políticas públicas. UPEL, 12 (1), 118-139

Glickman, C. (1990). Supervision of instruction. A devepelomental approach. Second edition. Massachusets: Ally and Bacon

González, E. (2007). Un Modelo de Supervisión Educativa. Laurus Revista de Educación 13 (25), 12-35. Recuperado de http://www.redalyc.org/pdf/761/76111 479002.pdf

Guarderas; W. (1993). La supervisión interna de instituciones educativas: Guía técnica. Quito; Ecuador: Limusa

Hernández, R; Fernández, C; y Baptista. (2010). Metodología de la investigación (5 ${ }^{\text {a }}$ ed.).México: McGraw-Hill/Interamericana

Husserl, H. (1974). Ideas relativas a una fenomenología pura y una filosofía fenomenológica. México: Fondo de Cultura Económica

Kaufman, R. (1976). Planificación de los sistemas educativos. México. D.F. Editorial Trillas

Martínez, M. (2012). Comportamiento humano. Nuevos métodos de investigación 2da edición. México: 1996 (reimpresión 2011). ISBN 978-968-24-5503-2 
Orozco, J. (2009). ¿Calidad de Educación o Educación de Calidad? Una preocupación más allá del Mercado. Revista Iberoamericana de Educación. $N^{\circ} 51,161$ 181. Consultado: 4/01/2016. Recuperado de http://www.rieoei.org/rie51a08.pdf

Pozner, P. (2000). Competencias para la profesionalización de la gestión educativa. Módulo 2. Buenos Aires, IIPE-UNESCO, 1617

Rojas, B. (2014). Investigación Cualitativa. Fundamentos y Praxis. $3^{\text {era }}$ Edición. Fondo Editorial de la Universidad Pedagógica Experimental Libertador (FEDUPEL). Caracas, Venezuela

Sánchez, J. (2013). Paradigmas de investigación educativa: De las leyes subyacentes a la modernidad reflexiva. Revista Interdisciplinar Entelequia, 16, 91103. Recuperado de https:// revistaentelequia.wordpress.com/2013/1 $0 / 12 / 1320 /$

Secadura, T, (2008). Principios de la supervisión educativa en el estado español: fortalezas y debilidades. Avances en supervisión educativa (8), 1-14. Recuperado de https://avances.adide. org/index.php

Soler, E. (2001). La supervisión educativa en sus fuentes. Madrid. Santillana.

Soler, E. (2015). Decálogo de las competencias profesionales del inspector de educación. Revista iberoamericana sobre calidad, eficacia y cambio en educación, 13, (4), 149-160. Recuperado de http://www.rinace.net/reice/

Strauss, A. y Corbin, J. (2002). Bases Cualitativas de la Investigación. Técnicas y Procedimientos para desarrollar la Teoría Fundamentada. Editorial Universidad de Antioquia.

Stufflebeam, D. (1996). El papel de la evaluación en la mejora escolar. El Gran cuadro. Bilbao. España. Ediciones Mensajero, S.A

Tamayo, A; y Valiente, P. (2019). El principio de la unidad de orientación, la educación que queremos para la generación de los bicentenarios. Recuperado de http://www.oei.es/ metas2021.pdf 
integralidad y el enfoque pedagógico de la supervisión educativa. En Transformación 15(1), 129-146. Recuperado de http://scielo.sld.cu/scielo.php?script=sci_ arttext\&pid=S2077$29552019000100129 \& \operatorname{lng}=\mathrm{es} \& \operatorname{tn} \mathrm{l}=\mathrm{es}$

Teppa, S. (2012). Análisis de la información cualitativa y construcción de teorías. Barquisimeto-Venezuela. Ediciones GEMA, C.A

Texeidó, M. (1997). Supervisión del sistema educativo. Barcelona, España. Editorial Ariel. S.A

Topete, C; Chávez, M; Márquez, C. (s.f). Desafíos en la formación del supervisor escolar como gestor de comunidades académicas en la sociedad del conocimiento. En X Congreso Nacional de Investigación Educativa, Área 13: política y gestión. 1-15. Recuperado de http://www.comie.org.mx/congreso/me moriaelectronica/v10/pdf/area_tematica_ 13/ponencias/1671-F.pdf

UNESCO. (2014). Informe de Seguimiento de la EPT (Educación Para Todos) en el Mundo. Enseñanza y aprendizaje: lograr calidad para todos. Recuperado de http://unesdoc.unesco.org/images/0022/ 002261/226159s.pdf

Valle, A; Castro, P; Reynoso, C; I, M; Stuart; y Duran, A. (2006). Dirección organización e higiene escolar. La Habana, Cuba: Pueblo y Educación

Vasilachis, I. (2006). Estrategias de Investigación Cualitativa. Editorial Gedisa S.A

Vélez, F. (2001). Cambios de paradigma en el desempeño directivo educacional. Caracas: Editorial Panapo 